\title{
What Can Universities Learn From Organizational Creative Space Design Research? A Look at Maker Spaces
}

\author{
Anne F. Santos \\ East Carolina University \\ Dennis Barber, III \\ East Carolina University \\ Michael Harris \\ East Carolina University \\ John Haymore \\ Lynn University
}

\begin{abstract}
The maker movement is a relatively nascent concept characterized by open access to resources and tools to foster innovation and creativity. This movement has created an opportunity for universities to support student ingenuity and originality. Organizational design and creativity research has provided conceptual frameworks and assessments to inform the physical layout of a maker space. This paper provides university leaders with propositions and processes for designing and assessing the impact of a maker space. The propositions are supported by theoretical models and assessment approaches. A central theme to the propositions is that the physical attributes of a maker space must be user centric.
\end{abstract}

Keywords: maker spaces, creativity, innovation spaces, entrepreneurship education

\section{INTRODUCTION}

The maker movement is a relatively nascent concept characterized by open access to resources and tools to foster innovation and creativity. Sparse empirical research on the movement exists, and the data and information which does exist often comes from secondary sources or periodicals. Dale Dougherty, founder of Make magazine, and the creator of the Maker Faire maker movement event, is often credited with stoking the fires of the modern maker movement as we know it. Dougherty (2012) states that the term maker is universal and applies to every person: "describing each one of us, no matter how we live our lives or what our goals might be" (p. 11). Chris Anderson, former editor-in-chief of Wired, distinguished the movement as the "new Industrial Revolution" (Anderson, 2012). Anderson states that "the Web has democratized the tools both of invention and of production," allowing anyone to be an entrepreneur and changing the entire future of manufacturing $(2012, \mathrm{p}$. 7). This democratization of resources has become a focal point of the movement. Due to decreasing resource costs for innovation in the form of low-cost or 
open source software as well as the fostering of maker spaces that give access to tools and equipment, people now more than ever have the ability to create and experiment (von Hippel, 2005). The maker movement provides a creative opportunity for entrepreneurs and students to collaborate in learning and knowledge sharing while using technological resources to create innovative products and services (Browder, Aldrich, \& Bradley, 2019).

The maker movement's effect on education and learning has largely been examined through the lens of constructivism and experiential learning theory (Martinez \& Stager, 2013). Constructivism is based on the notion of "mental construction," where cognitive development is characterized by reconciling new experiences with old via experimentation and exploration of concepts (Bada \& Olusegun, 2015). This construction is an active process: learners note elements of new learning experiences, apply their understandings, judge the consistency of the experience with prior knowledge, and modify their knowledge based on the experience (Phillips, 1995). Experiential learning theory follows in a similar vein with the learner being active in the process while focusing on exploration and doing. Experiential learning has been described as a process in which the student learns by doing where he or she can test hypotheses in the laboratory of real life (Dewey, 1971). Experiential learning has also been described as a holistic process that focuses on adapting to the learning environment (Kolb \& Kolb, 2005).

The purpose of this manuscript is to provide universities with maker spaces or intending to design such a space with a roadmap for how organizations have intentionally altered and used the physical environment to enhance creativity and innovation. Creativity and innovation will be used interchangeably throughout this paper. These suggestions are based on research findings of academics and practitioners working with how the environment can help or hinder creative behaviors in the workplace. This paper contains a review of the literature on how creativity has been addressed in the workplace focusing on how the social and physical environments have been assessed and altered to enhance creativity; a review of assessments, models, and frameworks recommended as tools that if applied can enhance creativity; propositions for maker space design and discussion of key elements; and concluding remarks and a call for action for university leaders.

\section{BACKGROUND}

\section{Maker Spaces in Universities}

Maker spaces are often associated with private industry. Although, universities have recently opted for the development of innovation spaces, fab labs, maker spaces, living lab, etc. (Delgado, Galvez, Hassan, Palominos, \& Morel, 2020). Delgado, et al. (2020) note that the focus on education and learning is an important distinction between company and university spaces. University maker spaces provide an environment for collaborative learning and reinforce the teaching-learning process. Wong \& Partridge (2016) observed maker spaces in $28 \%$ of observed Australian universities. These spaces typically employed specialized staff, provided specialized equipment such as laser cutters and 3D printers, and provided space to conduct coursework and collaborative projects (Wong \& Partridge, 2016). Pop-up maker spaces, temporary spaces brought to universities and libraries, have also been employed in several institutions (Wong \& Partridge, 2016; Lotts, Low-Cost High-Impact Makerspaces at the Rutgers University Art Library, 2017). These spaces are exploratory and allow institutions to gauge the performance and feasibility of maker spaces before deciding to implement permanent ones. Those that do implement permanent maker spaces have the advantage of tailoring the space to certain disciplines of interest (Xi, Wu, \& Zhang, 2017). The maker movement enhances student and entrepreneurial opportunities to extend the lifespan of products while addressing environmental challenges (Unterfrauner, Shao, Hofer, \& Fabian, 2019).

Maker spaces have also been prevalent in community education institutions such as public libraries. Library maker spaces hold promise for the democratization of innovation, described earlier, since they are typically free, community-oriented organizations (Halverson \& Sheridan, 2014). These library spaces have been praised as being affordable, taking up little space, and allowing libraries to form connections and partnerships within the community (Lotts, 2016). The main concerns associated with these spaces is the staffing of them, the messiness of the materials involved, and the safety of any specialty equipment provided 
(Lotts, 2016). These benefits and concerns would likely translate to other public education institutions, such as universities, as well.

\section{Creativity and Workplace Environment}

Researchers studying creative behaviors study it as creativity, innovation, and/or imagination. In the research literature, creativity, innovation, and imagination have similar underlying concepts with important distinctions. Creativity studies, largely in psychology, define their subject of research by saying that it has something to do with original or novel work, that the outcome is useful, adaptive, or effective, and a realistic solution for the problem being tackled. Some argue for adding the criterion of surprise to the standard definition of creativity (Zhou \& Hoever, 2014). Creativity research in the field of organizational psychology tends to define creativity as an outcome that is both useful and novel to the organization (Zhou \& Hoever, 2014). Innovation is simply defined as the successful implementation of creative ideas by organizations and is studied mainly in Business and Organizational Psychology. Imagination "refers to mental representations of things or ideas not immediately present to the senses" (Forgeard \& Kaufman, 2016, p. 251) and is mainly studied by researchers in the field of education.

Regardless of how researchers approach creativity, innovation, and/or imagination, most agree that creativity is a positive phenomenon and that any organization greatly benefits when employees engage in creative cognitive processes and behaviors to innovate and improve products, services, and/or methods. When all employees routinely work to find better methods for improving daily challenges that arise from changing circumstances, organizations are better off. Furthermore, in dynamic environments, creativity is a vital process for businesses to thrive (Zhou \& Hoever, 2014). Dul and Ceylan (2011) wrote that, "all employees in an organization can produce novel and potential useful ideas" (p. 13) that can greatly benefit any organization. There is always room for improvement and fostering creativity in employees is the best way to discover how a service, process, or product could be changed for a company to grow and increase performance. In addition, creative work environments can advance employee well-being, increase job satisfaction, and lower intentions to leave (Dul \& Ceylan, 2011).

A large portion of researchers studying creativity have focused on how individual characteristics are related to creative performance. Individual characteristics, such as tolerance for ambiguity, openness to new experiences, and divergent thinking, have been linked to creative performance (Dul \& Ceylan, 2011). Thus, organizations wishing to increase creativity have typically relied more on selection of employees who possess these individual characteristics and less on creativity training and/or contextual factors as important elements that can increase or hinder employee creativity. However, Zhou \& Hoever (2014) warn that relying on selection to promote creativity may not have the intended results, as organizational context must support creative potential.

The environmental context has long been acknowledged as an important factor involved in creativity performance. For example, Rhodes (1961) wrote an influential paper on creativity studies and identified four factors of creativity: person, product, process, and press. Press refers to the environment. He wrote about how these factors, although studied separately, only function together as a unit. Another researcher who included the role of the environment in creative processes was Robert Sternberg. He proposed the investment theory, which stated that creativity requires a convergence of six separate but related resources: intellectual abilities, knowledge, styles of thinking, personality, motivation, and environment (Sternberg, 2006).

Glăveanu (2014) suggested approaching creativity from a more "molecular" perspective. By that he meant not considering person, process, products, and environment as units of analysis, but instead focusing on the dynamic interrelations between elements of the system. He suggested using the Five A's Model (actors, audiences, actions, artifacts, and affordances), developed within cultural psychology by Rhodes (1961), to study creativity. Glăveanu called for approaching creativity as a complex process with shifting boundaries instead of as a single and separate construct. Using this model blurs the lines between person, product, and process allowing for a more realistic understanding of the complex phenomenon that is creativity. Thus, the environment has been recognized as an important aspect influencing employee creativity performance. 
In the innovation management literature, divergent thinking refers to expansive and explorative thinking which occurs in the initial phases of idea generation by meandering forward and in so doing, increasing dimensions and complexity. On the other hand, convergent thinking occurs in the validation phase and refers to integration processes that reduce complexity leading to a temporary state of balance of the system of ideas being worked on. Haner (2005) argued that successful innovation and creativity processes depend on an informal mixture of mastering convergence, divergence, and the transition between the two. Another dimension Haner (2005) discussed as important for organizations to provide support through physical spaces is to balance individual work and teamwork. Based on these findings, he proposed a model, which is presented in a later section of this paper.

Even though there is a large amount of anecdotal evidence supporting the importance of the role that the physical environment plays in enhancing creative behaviors, it has rarely been studied more systematically (Moultrie, et al., 2007). How the physical work environment supports or hinders creativity has been more widely researched in fields such as environmental psychology, architecture, interior design, and ergonomics (Dul \& Ceylan, 2011). When Glăveanu (2014) outlined some of the main models suggested and used by scholars of creativity, he concluded that the physical environment has been the most neglected. The propositions put forth in this paper directly address the physical design of the maker space.

Oksanen and Ståhle (2013) investigated how physical spaces and innovation interact with each other and identified important aspects of the physical environment that matter most for innovation. They characterize innovation as a process that is both communicative and human-centered (Oksanen \& Ståhle, 2013). These authors wrote that research on the physical environment and innovation is fragmented and theoretical foundations are limited. Whereas global trends are allowing for a new knowledge to emerge between people working together in different places, tacit knowledge can only emerge in physical spaces and is only available to people present in the physical space (Oksanen \& Ståhle, 2013). These authors support the notion that the physical environment is a required source for creativity and innovation. Based on the narratives collected from literature, interviews, and in-depth discussions with experts, developers, pioneers, and users of creative, innovative spaces, and learning environments; the authors proposed five attributes of the physical environment which enhance innovation: collaboration enabling, modifiability, smartness, attractiveness, and value reflecting. The implication is not that these elements certainly produce more innovations, but that a physical space supports the changing nature of innovation.

One of the main principles guiding how to create innovation spaces is that creativity is a social process, and the space should somehow enable and enhance this fact. More private spaces should also be made available for individual work because spaces that enable communication and teamwork hinder individual creative projects. It is important to note that elements of innovation are changing and becoming more versatile to include values such as sustainability and openness and these intersect with attributes of innovation spaces. For example, IDEO, a design company, values creativity, open mindedness, collaboration, and playfulness, and their physical workspaces reflect and enhance these values with a variety of spaces such as rooms for projects, open studios, a prototyping workshop, and a community garden. From a strategic perspective, the physical characteristics of the environment should relate to the strategic goals of the organization (Oksanen \& Ståhle, 2013). Designing innovative spaces requires detailed investigations of the cultural context and what users believe and value. More research is needed on physical spaces that can change the general rules of the innovation game with factors such as the socio-political context, market demands, competitor and customer behaviors, and technological possibilities.

Organizations with employees engaging in creative behaviors are better off than organizations with employees not engaging in creative behaviors. The physical environment is acknowledged as being one of the key components to foster creative behaviors in all employees. If the physical environment (in comparison to the social environment) is perhaps the most easily manipulated by organizations, then how can universities use these findings to better design maker spaces? 


\section{MODELS AND FRAMEWORK}

Informed by research on and experience in workplace creative spaces, researchers and practitioners have suggested different models and frameworks that can be used to assess information related to how the environment can promote and enhance creative behaviors in the workplace. These provide useful ways in which organizations can better understand where efforts can be placed to alter the physical (in support of efforts to alter and maintain the social) environment in ways that have shown to enhance creative behaviors. One of the benefits of applying assessments, models, and frameworks is that they allow for the unique needs of any one organization to be addressed. When applied to specific contexts, these tools can help organizations identify if smaller goals are in line with larger organizational goals and can point out the specific ways in which their physical work environment can be modified to achieve the most enhancement in creativity performance. This paper is meant as a literature review of assessments, models, and frameworks provided by academic researchers and practitioner researchers that placed emphasis on the physical environment working with creativity in the workplace. Universities are encouraged to review these and to decide which one(s) would be the most useful if applied to their specific context at the moment and to use the propositions and models presented to lead towards a better understanding of which aspects of the specific physical work environment could be intentionally modified to enhance creativity. An assessment (Dul \& Ceylan, 2011) and three models (Moultrie et al.'s Transformational Model, Bustamante et al.'s Update of Moultrie et al.'s Transformational Model, and Haner's Model) follow.

\section{Assessment}

Dul \& Ceylan (2011) proposed an assessment that organizations can use to identify areas of improvement and this makes for a sound starting point. The assessment included 21 elements of the work environment that are known to enhance creativity. The first nine are part of the social-organizational dimension and the remaining 11 items refer to the physical environment. The first nine referring to the social dimension of the environment are: challenging job, teamwork, task rotation, autonomy in job, coaching supervisor, creative goals, recognition of creative ideas, and incentives for creative results. The remaining 11 items refer to elements in the physical environment that can foster creativity and these are: furniture, indoor plants/flowers, calming colors, inspiring colors, privacy, window view to nature, any window view, quantity of light, daylight, indoor (physical) climate, positive sound, and positive smell.

The assessment is called Creativity Development Quick Scan (CDQS) and can be used to help organizations identify the changes in the environment that, if implemented, would have the most impact on employee creativity. The CDQS is administered to employees and analyzed at the individual, team, and organizational levels. Employees are asked to rate how much of each of the 21 elements are "present" or "realized" in their work environment. These scores added together represent the employee's perceived support received from their work environment. The CDQS also includes questions that ask how important each element is in supporting their creativity and provides an "importance" score. When the total "present" score is larger than the total "importance" score employees feel that their work environment supports their creativity. Higher "importance" scores than "present" scores indicate areas for improvement. Reiterating, organizations can administer this assessment to its employees as a first step to identify what changes in the environment would most enhance employee creativity behaviors.

\section{Moultrie et al. 's Transformational Model}

Informed by research and experience with creative spaces, an ongoing conversation started at the 'Creativity and Innovation Management' workshop held in 2005 in Oxford, England, Moultrie et al. (2007) proposed a transformational model. This model was meant as a framework for classifying and comparing the different designs of innovation environments.

Carefully considering the organization's strategic intent (input), what is involved in the process of creation (transformation), what the physical space offers (input), what is involved in the process of using the physical space (transformation), and what the realized intent is (output) allows organizations and 
researchers alike to consider each of these in the larger strategic and operational context of the organization. Figure 1 details a list of what organizations should consider for each of these aspects.

\section{FIGURE 1 \\ MOULTRIE ET AL.'S CONCEPTUAL FRAMEWORK}

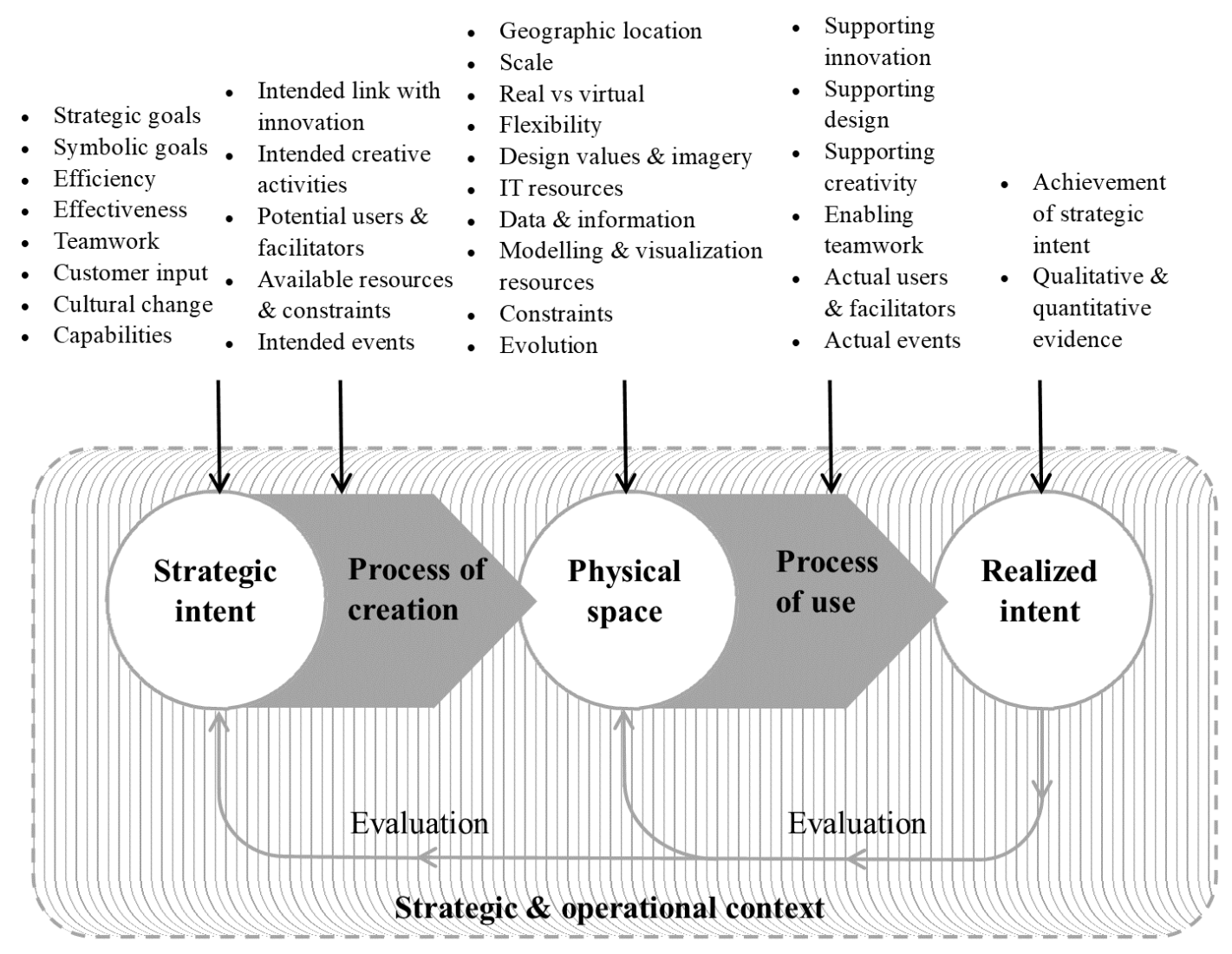

To apply this framework, organizations can begin by identifying both strategic and symbolic goals, as well as efficiency, effectiveness, teamwork, customer input, cultural change, and capabilities of the organization as it relates to strategic intent. The next step is to match the strategic intent to the process of innovation by considering the planned creative activities, possible users and facilitators, availability or scarcity of resources, and the intended events to take place in the creative space(s). The key in applying this model is to evaluate how an organization's strategic intent is reflected in the process of creation and how the needs in the process of use (of the physical space) are, or are not, met in the physical space. This process affords a clearer picture of where there may be discrepancies and, thus, where improvements can be maximized. One of the strengths of this model is that it accounts for what organizations with all types of creative spaces must address, such as deciding the importance of distinguishing between certain spaces (e.g., spaces used for creative activities and spaces used for training) and/or incorporating spaces not usually associated with creativity processes (e.g., spaces used for meetings including board meetings).

As such, this model considers factors relative to the process of creation, to the organization's strategic intent, and to the processes of use of the physical space and when applied provides a more comprehensive and systematic evaluation of how a physical space might or might not enhance creative behaviors. One of this model's strengths is that it includes the usage, creation, and intentions of the space (Moultrie, et al., 2007). 
Bustamante et al. 's Update of Moultrie et al. 's Transformational Model

Bustamante et al. (2015) proposed an updated version of this conceptual framework from which to better understand the influence of the physical space in the performance of innovation laboratories. Two of the authors working in the Lorrain Living Labs (located within the University of Lorraine in France) and two authors in the ViveLab Bogota (located within the National University of Colombia) approached the physical space as a conscious element of the environment that can enhance motivation to engage in innovative behaviors.

Several aspects of the physical environment are mentioned as being important to consider in spaces that motivate and enhance innovation. For example, findings show that innovation is most enhanced when the physical space supports participation by users, hinders hierarchy, allows for easy communication, and inspires play. In terms of how the organizational climate affects innovation, managers of spaces that foster creativity must steer away from methods that attempt to control behaviors and move towards enabling and cultivating techniques. They posit that the model proposed by Moultrie et al. (2007), with some updates, was a useful tool for researchers and practitioners wishing to help organizations create physical spaces that enhance their innovation processes. Bustamante et al. (2015) recommended the following updates to Moultrie et al.'s Transformational Model, which focused mainly on the strategic intention phase (see Figure 2).

FIGURE 2

\section{BUSTAMANTE ET AL.'S UPDATE TO MOULTRIE ET AL.'S TRANSFORMATIONAL MODEL}

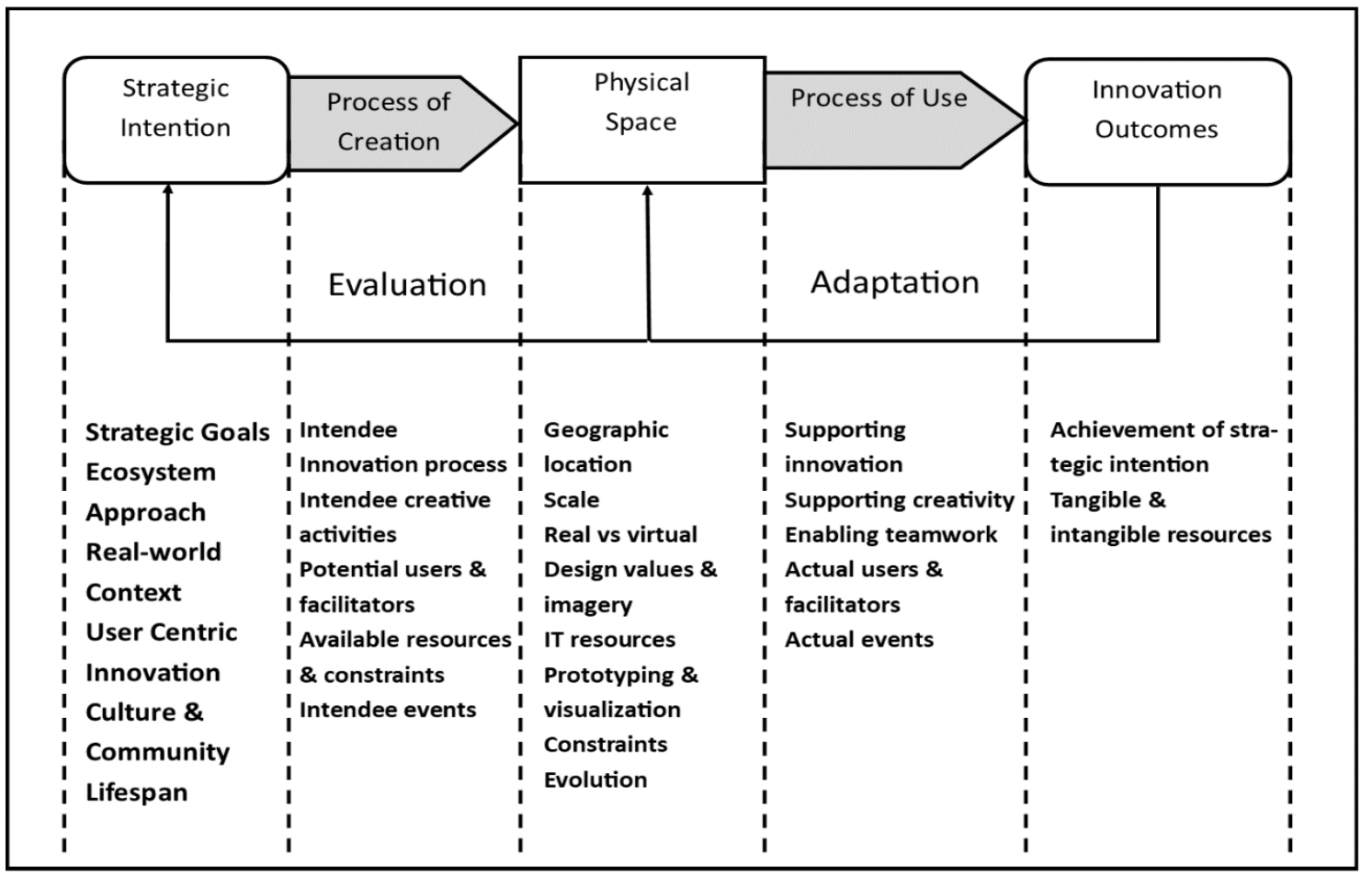

Bustamante et al. (2015) recommended keeping only strategic goals and teamwork from Moultrie et al.'s Transformational Model and adding: ecosystem approach, real-world context, user centric innovation, culture and community, and lifespan as aspects to be examined in the strategic intention phase. In the strategic intention phase, an ecosystems approach results in generating added value to all stakeholders. Real-world context refers to emulating the real world environment through space, equipment, or methodologies, while user centric innovation refers to including users in the process of creation where they 
can give valuable feedback at different stages of the innovation process. Culture and community refers to working towards creating and maintaining a community of users who are engaged and motivated to use the space. The final addition, lifetime, refers to considering the time span that the project is expected to last. Realized intent refers to the achievement of the strategic intent and the evidence (quantitative and qualitative) of this achievement.

\section{Haner's Model}

Haner (2005) proposed a model and compared two innovation laboratories in well-established organizations: the Interactive Creativity Landscape office (part of the Fraunhofer Office Innovation Center) in the city of Stuttgart, Germany, and the Learning Garden (in a financial institution) in Scandinavia.

Hanner (2005) wrote that for a space to support convergent and divergent thinking, as well as individual and collaborative work must be addressed. The author distinguished between style/location and building/layout levels of analysis which is the level used in his study. Location refers to how research has found that having a space for meeting face-to-face significantly enhances the innovation process. Style, not decoration, refers to whether the space is perceived as pleasant and attractive by its users. Some research has found evidence to support the importance of style in creative and innovation processes while others have not. Haner (2005) suggests that it may be the single most important consideration. The style of a work environment can symbolize innovation and signal creativity.

For a space to more fully support the innovation process as defined by the dimensions of convergent and divergent thinking as well as team and individual work, it must facilitate spontaneous face-to-face interaction while also providing users with the ability to engage in solo work. Building layouts can be designed or arranged so that group members are at least on the same floor, have communal spaces where members will run into each other throughout the day, spaces where they can meet as a group as needed, and individual spaces where they can retreat to engage in solo creative work.

The ICL in Germany is part of a non-territorial working environment (with a clean-desk policy) for several researchers. During its inception, the ICL in Germany recognized the need for different processes at different phases of the larger innovation project and intentionally created spaces to support these processes, which are referred to as zones of interaction, action, and retreat, together creating the interaction creativity landscape (see Figure 3).

FIGURE 3

\section{ICL ORIGINAL CONCEPT}

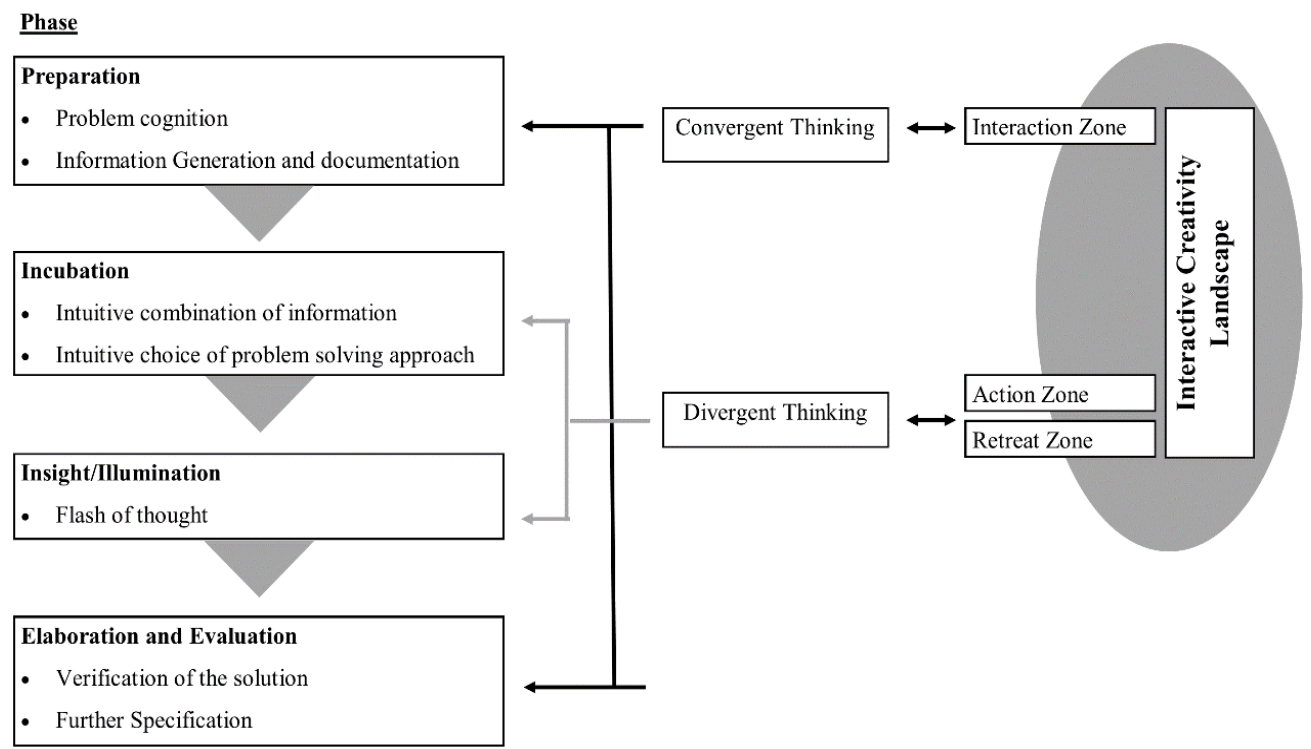

Source: (BAUER, HANNER, \& RIECK, 2001) 
The action zone supports divergent thinking, is the most spatially open part of the lab, contains the most support for communication retrieval and sharing, and is located near the communal area where people often engage in face-to-face informal communication. The interaction zone at the ICL called for more planning than the other zones because its aim was to support convergent thinking in more formal team efforts. The retreat zone was designed as a separated, individually adjustable space. The ICL treated soft factors such as colors, materials, and furnishings as important. However, the emphasis was on well-being and not necessarily comfort.

The other innovation lab analyzed by Haner (2005) was the Learning Garden in Scandinavia which had as its goal, during the planning phase, to enhance learning, creativity, and support for non-routine work processes. The lab has five different spaces each dedicated to a different process: the process arena, the exploration space, the creation garden, the consensus court, and the production studios. Employees move through phases of the process by physically moving through different spaces in the Learning Garden. The process arena is a round room with yellow as the main color designed to promote positive feelings (Haner, 2005). The exploration space provides support for communication and is a white, mostly blank, environment which invites ideas to be shared in small groups. The creation garden is where ideas are generated and proposed, and this space supports games that help this process. This space also supports easy documentation of the creative processes. The main color chosen for the creation garden was red to inspire stimulation and flexibility. The consensus court is where decisions are made by groups and this space has no regular seating for members, thus, requiring a higher level of planning prior to meeting in this space. Dark grey predominates this space inspiring neutrality and formality. The final space is the production studio where efficiency is activated, and decisions are refined and documented. Haner reported that both labs had become popular with their employees and were used for the intended purposes as well as for other meetings by other employees supporting other processes in the organization.

Haner (2005) discussed how these two innovation labs are similar and how they are different and provided a table summarizing his results as they relate to the dimensions of team and solo work as well as convergent and divergent processes (see Figure 4). Both the ICL and the Learning Garden were designed to support mainly creativity but also innovation and learning. Both provide varied infrastructures in the physical environment for employees to choose from as they move through a sequential model of the creative process. Designers of both labs paid close attention to color and materials in these spaces and chose according to the affect they sought to inspire during that phase. Also, both labs have spaces that allow for both divergent and convergent thinking processes. Convergent thinking occurs in ICL's interaction zone, while in the Learning Garden it occurs in the consensus court and the production studio. In the ICL, divergent thinking processes occur in the action and retreat zone, while in the Learning Garden they occur in the exploration space and the creative garden. The only space not easily designated for convergent or divergent thinking processes can be found in the Learning Garden's process arena and is more of an inbetween space (Haner, 2005). The differences between these labs are relative to the way that they are used by their employees.

\section{FIGURE 4}

\section{HANER'S MODEL COMPARING THE ICL AND THE LEARNING GARDEN}

\begin{tabular}{|c|c|c|}
\hline ICL & Divergence & Convergence \\
\hline Team & Yes & Yes \\
\hline Individual & Yes & Yes \\
\hline
\end{tabular}

\begin{tabular}{|c|c|c|}
\hline Learning Garden & Divergence & Convergence \\
\hline Team & Yes & Yes \\
\hline Individual & No & No \\
\hline
\end{tabular}

ICL is the domain of a specific research team, while the Learning Garden is used by a larger group of employees and this fact alone explains why the latter requires more planning for the use of the space while the other is open for use on a first-come basis. ICL's space was designed with flexibility in mind and 
provides users with a more seamless use of the space as they move between phases while the Learning Garden requires more co-planning. ICL provides opportunities for group and solo work, while the Learning Garden was designed for group use only. Given this usage pattern, ICL's physical space allows for employees to be visible and to have privacy providing mixed visibility options, while the Learning Garden provides high visibility and no privacy. The Learning Garden's physical space is well-structured and supports group cohesion among its users which enhances collaboration. The strength in ICL's physical space provides opportunities for self-organization without specific procedures in place.

In reviewing Haner's analysis of these two spaces built and used for creative endeavors, some conclusions emerge. Mainly that organizations wishing to use spatial design to enhance creative and innovative behaviors must tend to important principles including convergent and divergent thinking as well as solo and team work. The initial goals and actual usage, intentions, and outcomes, of the space must also be carefully examined to develop a deeper understanding of how the physical spaces can enhance creativity and innovation behaviors. In sum, this model provides a way to classify and organize the specific aspects of the physical space at any organization through the lens of four dimensions (divergent and convergent processes as well as team and solo work) known to influence how the physical space can support creativity performance in organizations. If an organization seeks to enhance employee creativity performance, they must have access to spaces that offer solo and team work capabilities as well as support divergent and convergent aspects of the process. Each organization can best define what those specific processes are for them and how the space could be altered to best support the needs arising from these processes. The previous review of literature and models provide the framework for actionable propositions for university leaders designing or managing maker spaces.

\section{PROPOSITIONS}

A central theme to the propositions is that the physical attributes of a maker space must be user-centric. With a focus on the end user, a space can generate the proper balance of structure with autonomy, interaction with seclusion, and comfort with usability. To ensure that a space is functioning as the designer and manager intended, a process of assessing the usage of the space will be valuable. There will be strategic goals for the space that may not be met when observing the use of the space over time. By beginning the building and design process through a user-centric lens, the team may be able to avoid a misalignment between actual use and strategic goals.

In the Bustamante et al. (2015) expansion of Moultrie et al.'s model (2007), user-centric innovation was added. It is highlighted that for a process of innovation to be user-centric, feedback is crucial. Within a university environment, academic activities and student collaboration must be considered. Students are the end users in university maker spaces and will use these spaces for creativity sessions, meetings and other user-centric activities (Delgado, Galvez, Hassan, Palominos, \& Morel, 2020). Also, having a usercentric process and focus can teach students the importance of user-centric design and innovation.

\section{Provide Individual Retreat Zones}

Workplace environments rarely provide support for both individual and group processes, meeting the need for collaboration and interaction while allowing for privacy when preferred (Haner, 2005). Even in an open office format, a quiet retreat zone is an important component to foster creativity and innovation. The physical environment influences creative work and protection from distraction and nurtures idea generation. A space which allows for the proper amount of interaction balances three dimensions: proximity, privacy and permission (Fayard \& Weeks, 2011). Private, individual retreat zones are conceptualized as a separated, cocoon-like space for users to experience quiet, isolation, and privacy (Haner, 2005).

A private space which encourages autonomy and reflection time are particularly important design factors to foster the creative process (Lukersmith \& Burgess-Limerick, 2013). Reflection in quiet spaces is important for students, and reflective thinking is a uniquely experienced phenomenon in learning (Patton $\&$ Kinsella, 2018). Students report at times they prefer to work at home when there is a lack of access to private spaces to conduct concentrated work (Katja Thoring \& Badke-Schaub, 2018). Integrating retreat 
zones also allows for a space to take a mental break, which start-ups have found to be critical for idea generation (Lee, 2016). This follows with previous research suggesting that incubation (at the individual level) is part of the creative process and happens through unconscious thought (Growe \& Mager, 2018). Private, personal spaces are where individuals conduct 'deep work' (Newport, 2016) including intense research, reading, writing or ideation activities (Katja Thoring \& Badke-Schaub, 2018).

\section{Create Spaces That Facilitate Spontaneous Interaction}

Historically, serendipity has been a misunderstood characteristic in the process of discovery and innovation (Martello, 1994). More recently, there has been an intentional focus on this quality and its importance for entrepreneurship. Collision, collaboration, and spontaneous interaction are all terms used in the literature to explain the same fundamental concept. Collaboration enabling is one of the attributes of a physical environment which fosters creativity and innovation (Oksanen \& Ståhle, 2013). Spontaneous interaction and collaboration happen when the environment is designed to encourage these collisions. Although, by nature, these interactions are random and unplanned, cannot be operationalized, and are triggered by unexpected encounters (Malmelin \& Virta, 2017). The use of common workspaces is valuable for creating a social infrastructure that spurs innovation and grows the economy (Smith, 2020).

Through social interaction, awareness emerges, enlightening ignorance, which would allow students to view their own knowledge gaps (Brinks, Ibert, Müller, \& Schmidt, 2018). The novel ways to view a problem or contextualize a situation provide a necessary condition for creativity and exploratory learning to ensure students create and not ossify (e Cunha, Clegg, \& Mendoça, 2010). Spontaneous interaction not only enhances the process of learning, but it is also an essential factor for team performance and product development (Sundström \& Zika-Viktorsson, 2009).

\section{Ensure the Space Is Modifiable and Comfortable}

The relationship between creativity and physical space is complex (Martens, 2011). Innovation and learning require flexibility. Innovation spaces are best when users can modify them according to their needs (Oksanen \& Ståhle, 2013). Flexible spaces (unfixed seating, moveable tables, white boards on wheels, etc.) are ideal for rapid prototyping and cross-pollination (Waber, Magnolfi, \& Lindsay, 2017), and physical spaces impact people's overall well-being (Samani, Abdul Rasid, \& bt Sofian, 2014). There has to be a balance, especially in open designs, between enabling informal interaction and avoiding distractions (Samani, Abdul Rasid, \& bt Sofian, 2014).

One of the issues with spaces that facilitate interactions, as discussed above, is that it sometimes is difficult to focus on individual work (Oksanen \& Ståhle, 2013). Modifiability aids in balancing the two first propositions. Modifiable designs bust silos, increase creativity, allow for brainstorming, and enhance iterative processes. Not only ought the space be flexible and modifiable in practice, but the aesthetic of flexibility plays a role. The spaces do not have to look like a showroom. Create a 'den' atmosphere as opposed to a 'parlor' (Becker, 2002) where comfort supports the flow of ideas (Oksanen \& Ståhle, 2013). This is a focus on style as opposed to decoration. A comfortable environment may lead to more intense sharing of ideas and increased engagement between students (Magadley \& Birdi, 2009).

\section{Include Artifacts}

Artifacts make up the first level of Schein's (1985) typology of organizational culture. Artifacts can be subtle, such as decision-making processes, or they can be directly observable like, for instance, physical space. Physical artifacts communicate visual data which stores and transfers organizational knowledge (Reischauer, 2015). Artifacts do not have to be static and can be used intentionally to create culture among students. Awareness of how to use cultural artifacts and the power they can possess directs the intentionality of physical space design. Sharing stories and sagas through artifacts and symbols builds culture and perpetuates strategic direction (Higgins, McAllaster, Certo, \& Gilbert, 2006). Cultural artifacts support innovation if they properly represent this as a strategic direction (Higgins \& McAllister, 2002) of the space for students. Tangible, physical artifacts are a form of knowledge and nurture the process of design and new product development (Narduzzo \& Lorenzoni, 2016). Organizational culture [therefore artifacts] 
deserves more attention because of its relationship to innovative orientation (Naranjo-Valencia, JiménezJiménez, \& Sanz-Valle, 2019) and student creativity.

\section{DISCUSSION}

This manuscript provides university leaders with specific tools that can be applied to assess, classify, and identify those aspects of the maker space environment that if modified would most enhance creativity, collaboration, and innovation. These tools were selected via a literature review of what scholars and practitioners in organizational design and innovation have been suggesting. The assessments, models, and frameworks mentioned here were selected because they were informed by an extensive literature review of the findings in the field and/or extensive experience working in the field.

In summary, Dul and Ceylan (2011) provided an assessment with 21 items (CDQS) that can be administered to employees resulting in scores that can help quickly identify what aspects of the environment, that if altered, could most impact employee creativity. This assessment can be analyzed at the individual, team, and organizational levels. Moultrie et al. (2007) suggested a transformational model where a list of aspects is to be examined under each stage of the process (input, transformation, and output) at both the strategic and operational level.

Bustamante et al. (2015) reviewed several models and concluded that Moultrie et al.'s model was by far the most useful if adapted with minor changes. The researchers recommend changing the aspects to consider in the strategic intentional phase as described above. By making these changes, they extended Moultrie et al.'s model, allowing organizations to offer creative spaces considering the time span that the project is expected to last (lifetime) and that support: generation of added value to all stakeholders (ecosystems approach), emulation of the real world environment (real-world context), inclusion of users in the process of creation where they can give valuable feedback at different stages of the innovation process (user centric innovation), as well as creation and maintenance of a community of users who are engaged and motivated to use the space (culture and community). Furthermore, he stated that realized intent should refer to the achievement of the strategic intent as well as the evidence (quantitative and qualitative) of this achievement. Reiterating, Bustamante et al. (2015) added that the realized intent phase should also be interpreted as an outcome of innovation and used to assess what might need to be adapted in the space and if strategic intent needs revisiting.

Haner (2005) offered a general framework from which to classify and assess innovative spaces, informed by research and experience that shows creative processes call for some combination of team and solo work as well as a combination of convergent and divergent processes. His work offered rich examples of two innovation labs and some of the details were included in the respective section above. The most useful aspect of this model is that it highlights these important dimensions to consider in efforts to enhance employee creativity, which allows organizations to identify if and what changes could be made towards enhancing employee innovation.

\section{CONCLUSIONS AND FUTURE RESEARCH}

This paper was written with the intent to provide those running or designing university maker spaces with an overview of the research examining the relationships between creativity and environment. More specifically, this paper provides research exploring how the physical environment could be altered to offer the greatest enhancement of creative behaviors, and to provide specific tools supported by scientific peerreviewed research and extensive field research findings. For example, the reader may choose to apply Dul and Ceylon's (2011) assessment if available institutional resources are scarce. Each model or framework highlights different aspects and offers a slightly different picture for future directions, yet all of them provide opportunities to appreciate the complexity and embeddedness of creative processes in larger institutional goals and practices as well as, once applied, directions in which universities can move towards further enhancing student creativity. 
Maker spaces are designed to encourage innovation and initiate entrepreneurial opportunities that can lead to business development. As discussed, two key aspects of any viable maker space are the right mixture of physical elements and social connections. The physical elements provide the backdrop for the social opportunities, thereby creating entrepreneurial collisions. These collisions happen due to proximity and design attributes and are necessary to create an innovative culture, particularly in a diverse university environment often marked by discipline specific silos. Future research could consider entrepreneurial behaviors such building high performance teams and sharing tools and technology with other collaborators and how policy can encourage economic growth through the utilization of the maker movement (Browder, Aldrich, \& Bradley, 2019).

Change is inevitable and universities must be able to transform its learning model to meet the complex and multifaceted disruptions that businesses face around the world (Purcell \& Chahine, 2019). Many universities claim to use entrepreneurship and innovation as means to bring their campuses together and break down traditional barriers. However, too often certain disciplines are overrepresented in entrepreneurial efforts, and maker spaces tend to cater to the needs of these specialties. Interdisciplinary teams impact student entrepreneurial attitudes and increase the likelihood of future entrepreneurial activities (Barber III, Madden, Mayo, \& Agarwala, 2020). And universities are unique in that most have such a diversity of disciplines, often a combination that is hard to replicate outside of an academic setting. The model provided by Bauer et al. (2001) highlights the important of convergent and divergent thinking and how space should be designed to allow for both.

In addition to the physical and social components, maker spaces should be an asset that builds and promotes an innovative culture and organizational processes within an organization. This type of culture ensures that entrepreneurial approaches are valued and rewarded within an organization. It also provides the foundation for authentic support from multiple disciplines across a university campus.

A distinct feature of a university campus is the concept of student learning. Most traditional students, particularly undergraduates, come to college with a desire to learn and a fundamental knowledge base, but lack the depth and breadth of knowledge to fully understand and embrace entrepreneurship and innovation. They often have the passion to tackle difficult challenges, but they need additional education and skill refinement to be impactful in their efforts. This educational component is a special feature of a university campus and maker spaces should be designed with this in mind. These young adults need enhanced learning opportunities to better understand and utilize a maker space and it creative tools.

Most successful entrepreneurial ventures are predicated on a team approach. Often, these teams are multidisciplinary and diverse in composition. Sometimes the team is developed through social or professional connections and other times through spontaneous interactions. As a part of its core function, a university maker space should create opportunities for cross-disciplinary collusions where entrepreneurial students are able to connect based on common themes but diverse skills. Too often, nascent entrepreneurs fall into the trap of believing they must possess all the necessary skills to start a new venture.

Future research should continue to explore the necessary elements for successful innovation spaces on college campuses. Many universities have these types of arrangements but too often they seem siloed and accessible by a limited number of university personnel. Perhaps more efficient hybrid models are needed to connect with existing resources and appeal to a broad group of faculty and students. The interdisciplinary nature of entrepreneurship and innovation requires constant collusions which lead to authentic engagement and the creation of diverse teams. These accommodations must be factored into a design that allows universities to move away from a maker space model to a dynamic innovation hub. 


\section{REFERENCES}

Anderson, C. (2012). Makers: The new industrial Revolution. New York: Crown.

Bada, S., \& Olusegun, S. (2015). Constructivism learning theory: A paradigm for teaching and learning. Journal of Research \& Method in Education, 5(6), 66-70.

Barber, D., III, Madden, L., Mayo, D., \& Agarwala, R. (2020). Two-course integration with student collaboration: Rapid prototyping and entrepreneurship. Entrepreneurship Education, 3, 57-76.

Bauer, W., Hanner, U-E., \& Rieck, A. (2001). OFFICE 21 ${ }^{\circledR}$ - Inventing an Interactive Creativity Landscape. In M. Smith, G. Salvendy, D. Harris, \& R. Koubek (Eds.), Proceedings HumanComputer-Interaction International Conference 2001 (volume 3, pp. 658-662). Mahwah: Lawrence Erlbaum Associates Publishers.

Becker, F. (2002). Improving Organisational Performance by Exploiting Workplace Flexibility. Journal of Facilities Management, 1(2), 154-162.

Brinks, V., Ibert, O., Müller, F.C., \& Schmidt, S. (2018). From Ignorance to Innovation: Serendipitous and purposeful mobility in creative processes - The cases of biotechnology, legal services and board games. Economy and Space, 50(8), 1742-1763.

Browder, R., Aldrich, H., \& Bradley, S. (2019). The emergence of the maker movement: Implications for entrepreneurship research. Journal of Business Venturing, (34), 459-476.

Bustamante, F.O., Reyes, J.I., Camargo, M., \& Dupont, L. (2015). Spaces to foster and sustain innovation: Towards a conceptual framework. 2015 IEEE International Conference on Engineering, Technology and Innovation/International Technology Management Conference (ICE/ITMC). Belfast.

Delgado, L., Galvez, D., Hassan, A., Palominos, P., \& Morel, L. (2020). Innovation Spaces in Universities: Support for Collaborative Learning. Journal of Innovation Economics \& Management, 31(1), 123-153.

Dewey, R. (1971). Accredited experiential education: Some definitions. The Urban and Social Change Review, pp. 10-15.

Dougherty, D. (2012). The Maker Movement. Innovation, 7(3), 11-14.

Dul, J., \& Ceylan, C. (2011). Work Environments for Employee Creativity. Ergonomics, 54(1), 12-20.

e Cunha, M.P., Clegg, S.R., \& Mendoça, S. (2010). On Serendipity and Organizing. European Management Journal, 28, 319-330.

Fayard, A-L., \& Weeks, J. (2011). Who Moved My Cube. Harvard Business Review, (July-August).

Forgeard, M.J., \& Kaufman, J.C. (2016). Who cares about imagination, creativity, and innovation, and why? A review. Psychology of Aesthetics, Creativity, and the Arts, 10(3), 250-269.

Glăveanu, V.P. (2014). On units of Analysis and Creativity Theory: Towards a "Molecular" Perspective. Journal for the Theory of Social Behaviour, 45(3), 311-330.

Growe, A., \& Mager, C. (2018). Changing Workplaces in the Creative Process in Creative Industries The case of advertising and Music. Erdkunde, 72(4), 259-272.

Halverson, E., \& Sheridan, K. (2014). The Maker Movement in Education. Harvard Education Review, $84(4)$.

Haner, U-E. (2005). Spaces for Creativity and Innovation in Two Established Organizations. Creativity and Innovation Management, 14(3), 288-298.

Higgins, J.M., \& McAllister, C. (2002). Want Innovation? Then use cultural artifacts that support it. Organizational Dynamics, 31(1), 74-84.

Higgins, J.M., McAllaster, C., Certo, S.C., \& Gilbert, J.P. (2006). Using Cultural Artifacts to Change and Perpetuate Strategy. Journal of Change Management, 6(4), 397-415.

Katja Thoring, P.D., \& Badke-Schaub, P. (2018). Creative Environments for Design Education and Practice: A typology of creative spaces. Design Spaces, 56, 54-83.

Kolb, A., \& Kolb, D. (2005). Learning styles and learning spaces: Enhancing experiential learning in higher education. Academy of Management Learning \& Education, pp. 193-212. 
Lee, Y.S. (2016). Creative Workplace Characteristics and Innovative Start-up Companies. Facilities, $34(7 / 8), 413-432$.

Lotts, M. (2016). Playing with LEGO ${ }^{\circ}$, Learning about the Library, \& "Making" Campus Connections: The Rutgers University Art Library Lego Playing Station, Part One. Journal of Library Administration, 56(4), 359-380.

Lotts, M. (2017). Low-Cost High-Impact Makerspaces at the Rutgers University Art Library. Journal of the Art Libraries Society of North America, 36(2), 346-362.

Lukersmith, S., \& Burgess-Limerick, R. (2013). The Perceived Importance and the Presence of Creative Potential in the Health Professional's Work Environment. Ergonomics, 56(6), 922-934.

Magadley, W., \& Birdi, K. (2009). Innovation Labs: An Examination into the Use of Physical Spaces to Enhance Organizational Creativity. Creativity and Innovation Management, 18(4), 315-325.

Malmelin, N., \& Virta, S. (2017). Organising Creative Interaction: Spontaneous and routinised spheres of team creativity. Communication Research and Practice, 3(4), 299-318.

Martello, W.E. (1994). Developing Creative Business Insights: Serendipity and its potential in entrepreneurship. Entrepreneurship \& Regional Development, 6, 239-258.

Martens, Y. (2011). Creative Workplace; Instrumental and symbolic support for creativity. Facilities, 29(1/2), 63-79.

Martinez, S., \& Stager, G. (2013). Invent to learn: Making, tinkering, and engineering in the classroom. Torrance, CA: Constructing Modern Knowledge Press.

Moultrie, J., Nilsson, M., Dissel, M., Haner, U-E., Janssen, S., \& Van der Lugt, R. (2007). Innovation Spaces: Towards a Framework for Understanding the Role of the Physical Environment in Innovation. Creativity and Innovation Management, 16(1), 53-65.

Naranjo-Valencia, J.C., Jiménez-Jiménez, D., \& Sanz-Valle, R. (2019). Organizational Culture Effect on Innovative Orientation. Management Decision, 49(1), 55-72.

Narduzzo, A., \& Lorenzoni, G. (2016). Physical Artifacts, Exaptation and Innovation as Novel Recombination. IFKAD Conference Proceedings.

Newport, C. (2016). Deep Work: Rules for focused success in a distracted world. London: Piatkus.

Oksanen, K., \& Ståhle, P. (2013). Physical environment as a source for innovation: Investigating the attributes of innovative space. Journal of Knowledge Management, 17(6), 815-827.

Patton, N., \& Kinsella, E.A. (2018). Reflective Spaces. In N. Patton, J. Higgs, \& M. Smith (Eds.), Developing Practice Capability (pp. 165-171). Leiden, The Netherlands: Brill | Sense.

Phillips, D. (1995). The Good, the Bad, and the Ugly: The Many Faces of Constructivism. Educational Researcher, 24(7), 5-12.

Purcell, W., \& Chahine, T. (2019). Leadership and governance frameworks driving transformational change in an entrepreneurial UK university. Leadership and Organization Development Journal, 40(5), 612-623.

Reischauer, G. (2015). Combining Artefact Analysis, Interview and Participant Observation to Study the Organizational Sensemaking of Knowledge-Based Innovation. Historical Social Research / Historische Sozialforschung, 40(3), 279-298.

Rhodes, M. (1961). An Analysis of Creativity. The Phi Delta Kappan, 42(7), 305-310.

Samani, S.A., Abdul Rasid, S.B., \& bt Sofian, S. (2014). A Workplace to Support Creativity. Industrial Engineering \& Management Systems, 13(4), 414-420.

Schein, E.H. (1985). Organizational Culture and Leadership. London: Jossey Bass.

Smith, T. (2020). Stand back and watch up: Post-capitalist practices in the maker movement. EPA: Economy and Space, 52(3), 593-610.

Sternberg, R.J. (2006). The Nature of Creativity. Creativity Research Journal, 18(1), 87-98.

Sundström, P., \& Zika-Viktorsson, A. (2009). Organizing for Innovation in a Product Development Project: Combining innovative and result oriented ways of working - A case study. International Journal of Project Management, 27(8), 745-753. 
Unterfrauner, E., Shao, J., Hofer, M., \& Fabian, C. (2019). The environmental value and impact of the maker movement - Insights from a cross-case analysis of European maker initiatives. Business Strategy and the Environment, 28(8), 1518-1533.

von Hippel, E. (2005). Democratizing innovation: The evolving phenomenon of user innovation. Management Review Quarterly, 55(1), 63-78.

Waber, B., Magnolfi, J., \& Lindsay, G. (2017). Workspaces That Move People. Harvard Business Review.

Wong, A., \& Partridge, H. (2016). Making as Learning: Makerspaces in Universities. Australian Academic and Research Libraries, 47(3), 143-159.

Xi, Q., Wu, W., \& Zhang, Y. (2017). Makerspace Activities in China. Science and Technology Libraries, $36(4), 425-433$.

Zhou, J., \& Hoever, I.J. (2014). Research on Workplace Creativity: A Review and Redirection. Annual Review of Organizational Psychology and Organizational Behavior, 1, 333-359. 